\title{
Methods
}

\section{Chemical Proteomics}

Synthesis of affinity matrix. Preparation of affinity matrix was performed as previously described. ${ }^{1}$ DN02 and Cmpd 1 were coupled at a density of 1 or $2 \mu \mathrm{mol}$ of compound per mL of NHSactivated sepharose beads, respectively.

Lysate generation. THP-1 cell pellets were suspended in lysis buffer (50 mM HEPES pH 7.5, $150 \mathrm{mM}$ $\mathrm{NaCl}, 1.5 \mathrm{mM} \mathrm{MgCl} 2,1 \mathrm{mM}$ DTT, $0.8 \% \mathrm{NP}-40,5 \%$ glycerol, and 1x protease inhibitors (Thermo)) at 2x pellet volume. The samples were sonicated on ice for 30 seconds at $20 \%$ amplitude with a 2 second pulse ON and 3 second pulse OFF. Cell debris was removed by centrifugation at $20,000 \times \mathrm{g}$ for 20 minutes at $4^{\circ} \mathrm{C}$. Protein concentration was determined by BCA (Pierce) and diluted down to $5 \mathrm{mg} / \mathrm{mL}$ with ChemProt buffer (50 mM HEPES pH 7.5, $150 \mathrm{mM} \mathrm{NaCl}, 1.5 \mathrm{mM} \mathrm{MgCl}$, $1 \mathrm{mM}$ DTT, 0.4\% NP-40, 1x protease inhibitors (Thermo)).

Chemical proteomic experiments. $1 \mathrm{~mL}$ of $5 \mathrm{mg} / \mathrm{mL}$ THP-1 lysates was treated with competitor Cmpd 1 at a single concentration $(100 \mu \mathrm{M})$ or DN01 in a concentration-dependent fashion $(50,10,1,0.1,0 \mu \mathrm{M})$ for $1 \mathrm{~h}$ at $4^{\circ} \mathrm{C}$. Following incubation with competitor compounds, the lysates were incubated with $35 \mu \mathrm{L}$ affinity matrix comprised of immobilized Cmpd 1 or immobilized DN02 at $4^{\circ} \mathrm{C}$ for 4 or $2 \mathrm{~h}$, respectively. Proteins bound to affinity matrix were washed and eluted with LDS sample buffer (NuPAGE, Invitrogen) containing $10 \mathrm{mM}$ DTT, alkylated with $25 \mathrm{mM}$ iodoacetamide, and processed through detergent removal columns (Thermo). In-solution digestion and isobaric labeling with TMT reagents was performed according to standard procedures.

Quantitative proteomics by two-dimensional nano liquid chromatography-tandem mass spectrometry (nLC-MS/MS). Quantitative proteomics was performed as previously described. ${ }^{2}$ The TMTlabeled sample was reconstituted in $0.1 \%$ formic acid (FA) / $2 \%$ acetonitrile for offline high-pH reversed phase separation (RP10) using a Dionex UltiMate 3000 high-performance liquid chromatography (HPLC) system with fraction collection using Chromeleon v.6.8 (Thermo) software. Separation was achieved on an Xbridge C18 $3.5 \mu \mathrm{m} \mathrm{2.1 \times 150} \mathrm{mm} \mathrm{HPLC} \mathrm{column} \mathrm{(Waters)} \mathrm{using} \mathrm{a} \mathrm{flow} \mathrm{rate} \mathrm{of} 250 \mu \mathrm{L} \mathrm{min}{ }^{-1}$ and the following ternary gradient and conditions: mobile phase $A=$ water (HPLC grade), mobile phase $B=$ acetonitrile (HPLC grade), and mobile phase $\mathrm{C}=200 \mathrm{mM}$ ammonium acetate, $\mathrm{pH} 10$. Mobile phase $\mathrm{C}$ is held at $10 \%$ throughout gradient. Starting conditions are $89 \% \mathrm{~A}$ and $1 \%$ mobile phase $\mathrm{B}$, ramping mobile phase $\mathrm{B}$ to $80 \%$ over $65 \mathrm{~min}$. Fractions were collected in 96-well plate at $100 \mathrm{sec}$ per well. Fractions were reduced down to 12 fractions, dried, and reconstituted in $0.1 \%$ FA / $2 \%$ acetonitrile for nLC-MS/MS. All data were acquired using a Orbitrap Fusion Lumos coupled to an Easy-nLC 1200 nanoflow liquid chromatograph operating at $300 \mathrm{~nL} \mathrm{~min}-1$. Peptides were cleaned up using a $1 \mathrm{~cm}$ online custom trap then eluted on a custom $20 \mathrm{~cm}$ laser pulled 75 um column with $\mathrm{C} 18$ resin. Peptides were quantified using the synchronous precursor selection (SPS-MS3) or higher-energy C-trap dissociation (HCD) method for TMT quantitation with 120k MS1 resolving power, $50 \mathrm{~ms}$ max injection time and 100\% automatic gain control (AGC). MS2 spectra were selected using the top ten most abundant features with a charge state between 2-6 using collision induced dissociation (CID) in the ion trap. The AGC was set to $100 \%$ with an isolation width of 0.7 . MS3 was performed on the top 10 most abundant MS2 features between 400-1600 amu with a collision energy of $55 \%$, AGC of $250 \%$ and $50 \mathrm{k}$ resolution. For HCD TMT quantitation the MS2 isolation width was set to 1.2 with a collision energy of $55 \%$, AGC of $250 \%$ and a resolution of $50 \mathrm{~K}$.

Mass spectrometry data processing. Peptide and protein identification and quantification were performed using Proteome Discoverer version 2.2 (Thermo). MS data were searched using the Mascot (Matrix Science) search engine against the UniProt reference database (human proteins, 42,233 entries, downloaded 2017) containing common contaminants and reversed sequences. Carbamidomethylated cysteine, oxidation of methionine, and TMT modification on $\mathrm{N}$-termini and lysine were set as dynamic modifications. Trypsin was specified as the proteolytic enzyme with up to one missed cleavage site allowed. Precursor and fragment ion tolerances were set to $10 \mathrm{ppm}$ and $0.8 \mathrm{Da}$, respectively. Search results were filtered for a minimum of 2 unique peptides, 1\% FDR peptide and protein identification and quantification, and common contaminants. Protein abundances were normalized and competition ratios were calculated using scripts provided by Proteome Discoverer. Dose-response curves were fitted to a three-parameter log logistic regression using GraphPad Prism. 


\section{Alphascreen}

BRD9(bd), BRD4(1), and BRD8(1) Ligand-Displacement AlphaScreen assays were performed using AlphaScreen Nickel Chelate Detection Kit reagents (Perkin Elmer) with the following specifications. Protein was prepared as previously described. ${ }^{3}$ Compound dilutions were prepared in DMSO in Echo Qualified 384-Well Low Dead Volume Microplates (Labcyte) and pre-transferred to 384-well plates (Proxiplate-384 White, PerkinElmer, USA) by acoustic transfer using Echo 500-series liquid handler. Alphascreen reagents were diluted in $50 \mathrm{mM}$ HEPES, $150 \mathrm{mM} \mathrm{NaCl}, 0.1 \% \mathrm{w} / \mathrm{v} \mathrm{BSA}, 0.01 \% \mathrm{w} / \mathrm{v}$ Tween20, and 1uM DTT, pH 7.5. A 2x solution of $6 \mathrm{x}$ His bromodomain and indicated tracer (concentrations below) was added at $10 \mu \mathrm{L} /$ well to compound-containing 384-well plates. Under low light, a 2x suspension of $\mathrm{Ni}$ coated Acceptor Beads and Streptavidin Donor Beads was then added at $10 \mu \mathrm{L} /$ well to a final concentration of $5 \mu \mathrm{g} / \mathrm{ml}$. The plates were spun down at $150 \mathrm{~g}$, incubated at room temperature protected from light for 1 hour, and read on an Envision 2104 (PerkinElmer, USA). Data were analyzed using GraphPad PRISM V8.

\begin{tabular}{|c|c|c|c|c|}
\hline Assay & Protein & [Protein] Final (nM) & Tracer & {$[$ Tracer] Final (nM) } \\
\hline BRD4 & 6xHis-BRD4(1) & 50 & Biotin-JQ1 & 10 \\
\hline BRD8 & $\begin{array}{c}\text { 6xHis-BRD8(1) } \\
\text { (Reaction Biology) }\end{array}$ & 30 & $\begin{array}{c}\text { Compound-1-Biotin } \\
\text { (synthetic proceedures) }\end{array}$ & 100 \\
\hline BRD9 & 6xHis-BRD9(bd) & 30 & Compound-1-Biotin & 20 \\
\hline
\end{tabular}

\section{NanoBRET In-Cell Engagement}

Nanoluc-BRD4(FL), Nanoluc-BRD9(BD1), or Nanoluc-BRD8(BD1) (Promega) were transfected into HEK293 cells using Fugene HD (Promega) at a ratio of $0.25 \mathrm{ug} / \mathrm{million}$ cells with $9 \mathrm{X}$ equivalents of carrier DNA. After 24 hours, cells were trypsinized, washed, and resuspended in Opti-MEM (without serum or phenol red) to a density of 200,000 cells $/ \mathrm{mL}$. A 20x DMSO solution of the NanoBRET tracer was transferred at $5 \mu \mathrm{L} /$ well into White, Non-Binding Surface (NBS) 96-well plates (Corning) using a BRAVO workstation (Agilent), followed by addition of $85 \mu \mathrm{L} /$ well of cell suspension.

\begin{tabular}{|c|c|c|c|}
\hline Assay & Construct & Tracer & [Tracer] Final (nM) \\
\hline BRD4 & Nluc-BRD4-FL & PBI6580 (Promega) & 60 \\
\hline BRD8 & Nluc-BRD8(1) & PBI6580 (Promega) & 2000 \\
\hline BRD9 & Nluc-BRD9(bd) & $\begin{array}{c}\text { Compound-3-Tracer } \\
\text { (synthetic procedures) }\end{array}$ & 500 \\
\hline
\end{tabular}

Competitor compounds were serially diluted in DMSO, and plated as 10X solutions in OPTI-MEM before transfer to assay plate at $10 \mu /$ well via BRAVO. After $2 \mathrm{hr}$ incubation $\left(37^{\circ} \mathrm{C}, 5 \% \mathrm{CO}_{2}\right)$, a $3 \mathrm{X}$ solution of complete NanoBRET Nano-Glo substrate (containing 1x Extracellular NanoLuc inhibitor) in OptiMEM was added at $50 \mu \mathrm{L} /$ well, and gently mixed on orbital shaker. After 3 minutes, plates were read on an Envision 2104 (PerkinEImer, USA). Data were analyzed using GraphPad PRISM V8.

\section{Modeling}

The 3D model of BRD8, in complex of BI-9536, was built by the homology modeling program Prime (Schrödinger Release 2016-1: Prime, Schrödinger). The previously solved x-ray co-crystal structure of BRD9/BI-9536 complex (PDB ID: 5F1H) ${ }^{4}$ was chosen as the template for homology modeling, due to its inclusion of the same ligand (BI-9536). The resulting complex structure was further minimized by the molecular mechanism program Macromodel (Schrödinger Release 2016-1: MacroModel, Schrödinger) for 500 steps, with the position constraints of all the residues that are more than $5 \AA$ away from the ligand (BI9536). This refined structure of BRD8/BI-9536 complex was then used as the template for structure-based design in this study, including by the molecular docking program Glide in its SP mode (Schrödinger Release 2016-1: Glide, Schrödinger). 


\section{$\underline{\text { References }}$}

1. Gower, C. M.; Thomas, J. R.; Harrington, E.; Murphy, J.; Chang, M. E.; Cornella-Taracido, I.; Jain, R. K.; Schirle, M.; Maly, D. J., Conversion of a Single Polypharmacological Agent into Selective Bivalent Inhibitors of Intracellular Kinase Activity. ACS Chem Biol 2016, 11 (1), 121-31.

2. Ross, N. T.; Lohmann, F.; Carbonneau, S.; Fazal, A.; Weihofen, W. A.; Gleim, S.; Salcius, M.; Sigoillot, F.; Henault, M.; Carl, S. H.; Rodriguez-Molina, J. B.; Miller, H. R.; Brittain, S. M.; Murphy, J.; Zambrowski, M.; Boynton, G.; Wang, Y.; Chen, A.; Molind, G. J.; Wilbertz, J. H.; Artus-Revel, C. G.; Jia, M.; Akinjiyan, F. A.; Turner, J.; Knehr, J.; Carbone, W.; Schuierer, S.; Reece-Hoyes, J. S.; Xie, K.; Saran, C.; Williams, E. T.; Roma, G.; Spencer, M.; Jenkins, J.; George, E. L.; Thomas, J. R.; Michaud, G.; Schirle, M.; Tallarico, J.; Passmore, L. A.; Chao, J. A.; Beckwith, R. E. J., CPSF3-dependent pre-mRNA processing as a druggable node in AML and Ewing's sarcoma. Nat Chem Biol 2020, 16 (1), 50-59.

3. Salcius, M.; Bauer, A. J.; Hao, Q.; Li, S.; Tutter, A.; Raphael, J.; Jahnke, W.; Rondeau, J.-M.; Bourgier, E.; Tallarico, J., SEC-TID: A label-free method for small-molecule target identification. Journal of biomolecular screening 2014, 19 (6), 917-927.

4. Martin, L. J.; Koegl, M.; Bader, G.; Cockcroft, X.-L.; Fedorov, O.; Fiegen, D.; Gerstberger, T.; Hofmann, M. H.; Hohmann, A. F.; Kessler, D.; Knapp, S.; Knesl, P.; Kornigg, S.; Müller, S.; Nar, H.; Rogers, C.; Rumpel, K.; Schaaf, O.; Steurer, S.; Tallant, C.; Vakoc, C. R.; Zeeb, M.; Zoephel, A.; Pearson, M.; Boehmelt, G.; Mcconnell, D., Structure-Based Design of an in Vivo Active Selective BRD9 Inhibitor. Journal of Medicinal Chemistry 2016, 59 (10), 4462-4475. 